\title{
SOME FIXED POINT THEOREMS
}

\author{
P. V. SUBRAHMANYAM and I. L. REILLY
}

(Received 20 August 1990)

Communicated by J. H. Rubinstein

\begin{abstract}
Banach's contraction principle guarantees the existence of a unique fixed point for any contractive selfmapping of a complete metric space. This paper considers generalizations of the completeness of the space and of the contractiveness of the mapping and shows that some recent extensions of Banach's theorem carry over to spaces whose topologies are generated by families of quasi-pseudometrics.
\end{abstract}

1991 Mathematics subject classification (Amer. Math. Soc.): Primary 47 H 10, 54 H 25; secondary 54 E 40.

Keywords and phrases: contraction, sequentially complete, quasi-gauge space, fixed point.

\section{Introduction}

The well-known theorem of Banach [1] concerning contractive self mappings on a complete metric space has been widely used in existence theorems in differential and integral equations. In line with recent work on fixed point theorems, this paper considers generalizations of the two hypotheses of completeness of the metric space and the contractive nature of the map in Banach's theorem. Specifically, our main object is to show that results of Edelstein [4] and [5], and Reich [8] can be generalized to the non-metric situation afforded by gauge and quasi-gauge spaces. In this sense, our work is a continuation and extension of that of Tan [13].

A non-negative real valued function $p$ on $X \times X$ with the properties that

The second author wishes to acknowledge the support of the University of Auckland Research Committee.

(C) 1992 Australian Mathematical Society 0263-6115/92 \$A2.00+0.00 
$p(x, x)=0, x \in X$, and $p(x, y) \leq p(x, z)+p(z, y), x, y, z \in X$, is called a quasi-pseudometric on the non-void set $X$. According to Reilly [9] a topological space $(X, \mathscr{T})$ is a quasi-gauge [gauge] space $(X, \mathscr{T}, \mathscr{P})$, or simply $(X, \mathscr{P})$, if there exists a family $\mathscr{P}$ of quasi-pseudometrics [pseudometrics] such that the open balls

$$
B(x, p, \varepsilon)=\{y \in X \mid p(x, y)<\varepsilon\}, \quad x \in X, p \in \mathscr{P}
$$

form a subbase for the topology $\mathscr{T}$. In a quasi-gauge space $(X, \mathscr{T}, \mathscr{P})$, the sequence $\left\{x_{n}\right\}$ is said to be a left [right] Cauchy sequence if for each $p \in \mathscr{P}$ and each $\varepsilon>0$ there is a point $x \in X$ and an integer $n_{0}$ such that $p\left(x, x_{n}\right)<\varepsilon\left[p\left(x_{n}, x\right)<\varepsilon\right], n>n_{0}$. If every left [right] Cauchy sequence in the space $(X, \mathscr{T}, \mathscr{P})$ converges to a limit in the topology $\mathscr{T}$, the space is said to be left [right] sequentially complete. If $p$ is a quasi-pseudometric on $X$ so is $p^{\prime}$ defined by $p^{\prime}(x, y)=p(y, x), x, y \in X$, and $p^{\prime}$ is called the conjugate of $p$. And $\left(X, \mathscr{T}^{\prime}, \mathscr{P}^{\prime}\right)$, where $\mathscr{P}^{\prime}=\left\{p^{\prime}: p \in \mathscr{P}\right\}$ and $\mathscr{T}^{\prime}$ is the topology with the balls $\left\{B\left(x, p^{\prime}, \varepsilon\right), x \in X, \varepsilon>0, p^{\prime} \in \mathscr{P}^{\prime}\right\}$ as a subbase, is a quasi-gauge space and is called the conjugate of $(X, \mathscr{T}, \mathscr{P})$. Reilly [10] used the term completeness for what we call left sequential completeness. Right sequential completeness is thus completeness of the conjugate space in the terminology of [10]. It is well to note that left sequential completeness and right sequential completeness are independent notions.

EXAMPLE 1. The space $X=\{1 / n: n \in \mathbb{N}\}$ with the quasi-gauge induced by the quasi-pseudometric $p$ defined as

$$
p(x, y)= \begin{cases}x-y & \text { if } x \geq y \\ 1 & \text { if } x<y\end{cases}
$$

is readily seen to be right sequentially complete. But the left Cauchy sequence $\{1 / n\}$ is not convergent in $X$. (Incidentally the operator $T$ defined on $X$ as $T(x)=1 / 3$ for $x \neq 1 / 4$ and $T(1 / 4)=1 / 5$ is a continuous right Banach operator [12, Definition 4] which is not a left Banach operator implying thereby that the dual version of [12, Theorem 1] is non-vacuous.)

We extend in the following formal definitions the corresponding concepts for metric spaces to quasi-gauge spaces.

DEFinition 1. In the quasi-gauge space $(X, \mathscr{P}), x \in X$, is said to be $\varepsilon$-chainable to $y \in X$ for some $\varepsilon>0$ if for each $p \in \mathscr{P}$, there exist a finite number of $z_{i} \in X, i=0,1, \ldots, n(p)-1, n(p)$ such that $z_{0}=x$, $z_{n(p)}=y$ and $p\left(z_{i}, z_{i+1}\right)<\varepsilon$ for $i=0,1, \ldots, n(p)-1$. And $(X, \mathscr{P})$ is said to be $\varepsilon$-chainable if for any $x, y \in X$, either $x$ is $\varepsilon$-chainable to $y$ or $y$ is $\varepsilon$-chainable to $x$.

DEFINITION 2. An operator $T$ on a quasi-gauge space $(X, \mathscr{P})$ into itself is said to be an $\varepsilon$-local left [right] weak contraction if for each $x \in X$ and 
$p \in \mathscr{P}$ there exist $\varepsilon$ and $\lambda(\varepsilon>0,0 \leq \lambda<1)$ such that for each $y$ in $B(x, p, \varepsilon)$ we have

$$
p(T(x), T(y)) \leq \lambda p(x, y) \quad[p(T(y), T(x)) \leq \lambda p(y, x)] .
$$

If $\varepsilon$ and $\lambda$ are independent of $x$ though not of $p, T$ is called an $(\varepsilon-\lambda)$ uniformly local left [right] weak contraction.

However, we employ a more general definition for our purposes.

Definition 3. An operator $T$ on a quasi-gauge space $(X, \mathscr{P})$ is called an $\varepsilon$-local left [right] asymptotic weak contraction (for a positive $\varepsilon$ ) if for each $x \in X, p \in \mathscr{P}$ and $y \in B(x, p, \varepsilon)$ there is a sequence of real numbers $\lambda_{k}(y, \varepsilon)$ (depending on $\left.x, p, y\right)$ such that $\sum_{k=1}^{\infty} \lambda_{k}(y, \varepsilon)<+\infty$ and $p\left(T^{k}(x), T^{k}(y)\right) \leq \lambda_{k}(y, \varepsilon) \quad\left[p\left(T^{k}(y), T^{k}(x)\right) \leq \lambda_{k}(y, \varepsilon)\right]$.

REMARK. A contraction on a metric space is evidently a uniformly local left and right contraction and a uniformly local left [right] weak contraction on a quasi-gauge space is a local left [right] asymptotic weak contraction.

\section{On the contractiveness of the operator}

Edelstein [4, Theorem 5.2] showed in the case of an $\varepsilon$-chainable metric space that any $(\varepsilon-\lambda)$ uniformly local contraction on the space has a fixed point. His result generalizes as follows to a quasi-gauge space. We omit the proof, which is based on the technique in Edelstein's proof.

THEOREM 1. Let $T$ be a continuous operator on a Hausdorff left [right] sequentially complete quasi-gauge space $(X, \mathscr{P})$ into itself such that

(i) $T$ is an $\varepsilon$-local left [right] asymptotic weak contraction,

(ii) for some point $x$ in $X, x$ is $\varepsilon$-chainable to $T(x)[T(x)$ is $\varepsilon$-chainable to $x$ ].

Then $T$ has a fixed point in $X$.

If $(X, \mathscr{P})$ is an $\varepsilon$-chainable sequentially complete Hausdorff gauge space then the hypothesis of continuity is redundant, since a local weak contraction is necessarily continuous. Also in this case the fixed point is unique.

We observe that the Hausdorff requirement in Theorem 1 can be weakened to the condition that convergent sequences have unique limits, that is, that the space be a $U S$ space in the sense of Wilansky [14].

The following example shows that even for metric spaces the theorem is stronger than that of Edelstein.

EXAMPLE 2. Let $X$ be a subset of the space of bounded real sequences with the supremum-norm defined by $X=\bigcup_{n=1}^{\infty} I_{n}$ where $I_{1}$ is the set $\left\{\alpha e_{1}: \alpha \in\right.$ 
$[0,2]\}$ and $I_{n}$ is the set $\left\{\alpha e_{n}: \alpha \in[1,2]\right\}$ for $n \geq 2, e_{n}$ being the sequence taking 1 at the $n$th place and elsewhere identically zero for each $n$. Let $T$ be the operator on $X$ defined by

$$
T(x)= \begin{cases}x / 2, & \text { if } x \in I_{1}, \\ \alpha e_{n-1}, & \text { if } x=\alpha e_{n}, n \geq 2 .\end{cases}
$$

It is clear that $X$ is complete in the induced metric, though not compact. Besides it is $\varepsilon$-chainable for $\varepsilon=3 / 2$. For any pair of elements $x \quad\left(=\alpha e_{n}\right)$ and $y\left(=\beta e_{m}\right)$ with $\|x-y\|<3 / 2$ it follows that $\left\|T^{k}(x)-T^{k}(y)\right\|<$ $1 / 2^{k-s-2}$ for all $k>s$, where $s=\max \{m, n\}$. So the continuous operator $T$ is an $\varepsilon$-local asymptotic weak contraction for $\varepsilon=3 / 2$. Hence by Theorem 1 , every sequence of iterates converges to a fixed point which is unique. By considering $T$ on $I_{n}$ (for $n \geq 2$ ) it follows that $T$ is not an $(\varepsilon-\lambda)$ uniform contraction, however small $\varepsilon$ may be. Thus Edelstein's theorem cannot be used in this case to establish the fixed point of $T$.

Next we generalize to quasi-gauge spaces a result of Reich [8, Proposition 1.2]. The hypotheses on the operator were motivated by the extension of the fixed point theorem of Kannan [6] given in Subrahmanyam [12, Theorem 2].

THEOREM 2. Let $T$ be an operator (not necessarily continuous) of the quasigauge space $(X, \mathscr{P})$ into itself. Suppose that

(i) $\mathscr{P}$ contains a conjugate pair $\left(p_{0}, p_{0}^{\prime}\right)$,

(ii) there exist non-negative real numbers $a, b, c, d$ and $e$ such that for $x \neq y$

$$
\begin{aligned}
p_{0}(T(x), T(y))< & a p_{0}(x, T(x))+b p_{0}(y, T(y))+c p_{0}(x, T(y)) \\
& +d p_{0}(y, T(x))+e p_{0}(x, y),
\end{aligned}
$$

(iii) $1>b+c$ and $(a+c+e) /(1-b-c)=1$,

(iv) $X$ is sequentially compact in the topology induced by $\mathscr{P}$.

Then $T$ has a fixed point. Further, if $d /(1-c-e) \leq 1$, the fixed point is unique.

Proof. Let $r$ be $\inf \left\{p_{0}(x, T(x)): x \in X\right\}$. Then we can find a sequence of points $x_{n}$ in $X$ such that $p_{0}\left(x_{n}, T\left(x_{n}\right)\right)$ converges to $r$. As $X$ is sequentially compact, $T\left(x_{n}\right)$ has a subsequence $y_{k}=T\left(x_{n k}\right)$ converging to $y$.

Since

$$
\begin{aligned}
p_{0}(y, T(y)) \leq & p_{0}\left(y, T\left(x_{n k}\right)\right)+p_{0}\left(T\left(x_{n k}\right), T(y)\right), \\
p_{0}\left(T\left(x_{n k}\right), T(y)\right) \leq & a p_{0}\left(x_{n k}, T\left(x_{n k}\right)\right)+b p_{0}(y, T(y))+c p_{0}\left(x_{n k}, T(y)\right) \\
& +d p_{0}\left(y, T\left(x_{n k}\right)\right)+e p_{0}\left(x_{n k}, y\right), \\
p_{0}\left(x_{n k}, T(y)\right) \leq & p_{0}\left(x_{n k}, T\left(x_{n k}\right)\right)+p_{0}\left(T\left(x_{n k}\right), y\right)+p_{0}(y, T(y)),
\end{aligned}
$$


and

$$
p_{0}\left(x_{n k}, y\right) \leq p_{0}\left(x_{n k}, T\left(x_{n k}\right)\right)+p_{0}\left(T\left(x_{n k}\right), y\right)
$$

we have that

$$
\begin{aligned}
p_{0}(y, T(y)) \leq & (a+c+e) p_{0}\left(x_{n k}, T\left(x_{n k}\right)\right)+(b+c) p_{0}(y, T(y)) \\
& +(1+d) p_{0}\left(y, T\left(x_{n k}\right)\right)+(c+e) p_{0}\left(T\left(x_{n k}\right), y\right) .
\end{aligned}
$$

Hence

$$
\begin{aligned}
(1-b-c) p_{0}(y, T(y)) \leq & (a+c+e) p_{0}\left(x_{n k}, T\left(x_{n k}\right)\right) \\
& +(1+d) p_{0}\left(y, T\left(x_{n k}\right)\right)+(c+e) p_{0}^{\prime}\left(y, T\left(x_{n k}\right)\right) .
\end{aligned}
$$

Proceeding to the limit as $k \rightarrow \infty$ we have $(1-b-c) p_{0}(y, T(y)) \leq$ $(a+c+e) r$. As $(a+c+e) /(1-b-c)=1$ it follows that $p_{0}(y, T(y))=r$. If $y \neq T(y)$, we obtain from (ii) that

$$
(1-b-c) p_{0}\left(T(y), T^{2}(y)\right)<(a+c+e) p_{0}(y, T(y)) .
$$

Hence $p_{0}\left(T(y), T^{2}(y)\right)<p_{0}(y, T(y))=r$, which contradicts the definition of $r$, so that $y=T(y)$ as desired.

If $x$ and $y$ are both fixed points of $T$, then

$$
p_{0}(x, y)=p_{0}(T(x), T(y))<(c+e) p_{0}(x, y)+d p_{0}(y, x)
$$

from (ii). Thus $(1-c-e) p_{0}(x, y)<d p_{0}(y, x)$. Similarly, interchanging the roles of $x$ and $y$ gives $(1-c-e) p_{0}(y, x)<d p_{0}(x, y)$. Hence

$$
(1-c-e)\left\{p_{0}(x, y)+p_{0}(y, x)\right\}<d\left\{p_{0}(y, x)+p_{0}(x, y)\right\},
$$

so if that $d /(1-c-e) \leq 1$ the fixed point is unique.

REMARKs. Reich's result mentioned above corresponds to the case $\mathscr{P}=$ $\{d\}, d$ a metric and $a=b=1 / 2, c=d=e=0$.

Condition (iii) in Theorem 2 can be replaced by the alternative

(iii) $^{\prime} 1>a+d$ and $(b+d+e) /(1-a-d)=1$.

\section{Convergence of iterates}

We begin by stating a result of Bailey [2] supplementing one of Edelstein [5, Theorem 1]: if $(X, d)$ is a compact metric space and $T$ is a weakly contractive continuous operator on it (that is, there exists an integer $n$ such that $d\left(T^{n}(x), T^{n}(y)\right)<d(x, y), x, y \in X$ and $n$ depends on $x$ and $\left.y\right)$, then $T$ has a unique fixed point. In passing, we remark that the assumption of continuity cannot be dispensed with in the above result. 
Example 3. Let $X=[0,1]$ and $T$ be given by $T(0)=1 / 2$ and $T(x)=$ $x / 2$ on $(0,1]$. Then $T$ is not continuous at 0 and has no fixed-point. But $T$ is weakly contractive over $[0,1]$. Indeed if $x, y$ are in $(0,1]$ then $|T(x)-T(y)|=|(x-y) / 2|<|x-y|$ for $x \neq y$. If $x=0$ and $y \in(0,1]$ then there exists $n \geq 1$ such that $|(1-y) / y|<2^{n}$. Then $\left.\mid T(x)-T(y)\right) \mid=$ $\left|(1-y) / 2^{n}\right|<y=|x-y|$.

With Bailey's result in the background the following theorem generalizes Edelstein's assertion that any contractive operator $T$ on a metric space with a convergent subsequence of $T$-iterates has a fixed point.

THEOREM 3. Let $T$ be a continuous operator on a quasi-gauge space $(X, \mathscr{P})$ into itself such that

(i) there exists a pseudometric $p_{0}$ in $\mathscr{P}$ such that

$$
p_{0}\left(T(x), T^{2}(x)\right) \leq p_{0}(x, T(x)), x \in X,
$$

(ii) for each $x \neq T(x)$, there is a positive integer $n$ such that

$$
p_{0}\left(T^{n}(x), T^{n+1}(x)\right)<p_{0}(x, T(x)),
$$

(iii) $x_{n k}=T^{n k}(x), k=0,1,2, \ldots$, a subsequence of $T$-iterates at some point $x$, converges to $u$.

Then $u$ is a fixed point of $T$.

Proof. Since $T$ is continuous and $p_{0}$ is symmetric the map $\phi_{T}(x)=$ $p_{0}(x, T(x))$ is a real valued continuous function on $X$. From (i) it is clear that $\left\{\phi_{T}\left(T^{n}(x)\right)\right\}$ is a monotonic decreasing sequence of non-negative real numbers. Hence this sequence converges to a real number $r$. As $x_{n k}$ converges to $u$ and $\phi_{T}$ is a continuous function, $\left\{\phi_{T}\left(x_{n k}\right)\right\}$ converges to $\phi_{T}(u)$ and so $\phi_{T}(u)=r$. If $u \neq T(u)$ then by (ii) there is a positive integer $m$ such that $\phi_{T}\left(T^{m}(u)\right)<\phi_{T}(u)$. But as $\left\{x_{n k}\right\}$ converges to $u,\left\{T^{m}\left(x_{n k}\right)\right\}$ converges to $T^{m}(u)$ due to the continuity of $T$. Hence $\left\{\phi_{T}\left(T^{m}\left(x_{n k}\right)\right)\right\}$ converges to $\phi_{T}\left(T^{m}(u)\right)$. Noting that the latter is a subsequence of $\left\{\phi_{T}\left(T^{n}(x)\right)\right\}$ we have $r=\phi_{T}(u)=\phi_{T}\left(T^{m}(u)\right)$, a contradiction of the choice of $m$. Hence $u$ is a fixed point of $T$.

COROLlary. Let $T$ be a continuous operator on a sequentially compact quasi-gauge space $(X, \mathscr{P})$ into itself such that there exists a fixed pseudometric $p_{0}$ in $\mathscr{P}$ with $p_{0}\left(T(x), T^{2}(x)\right)<p_{0}(x, T(x))$ whenever $x \neq T(x)$. Then $T$ has a fuxed point.

We observe that the use of the function $\phi_{T}$ as a measure of the expansive nature of $T$ was suggested by Dieudonné $[3$, p. 262, Problem 1]. 
In Theorem 3, the fixed point $u$ need not be unique. For example let

$$
X=\left\{(x, y):(x, y) \in \mathbb{R}^{2}, x^{2}+y^{2} \leq 1, x \geq 0, x^{2}+x \leq 1\right\} .
$$

Then the map $(x, y) \rightarrow\left(x^{2}, y\right)$ satisfies the conditions of Theorem 3 for the euclidean metric on $\mathbb{R}^{2}$ and has more than one fixed point.

EXAMPLE 4. Let $X$ be $[0,1]$ and $p$ be the pseudo-metric on $X$ defined by

$$
p(x, y)= \begin{cases}0 & \text { if } x, y \notin S, \\ 1 / 2^{k} & \text { if } x=1 / 2^{k} \text { and } y \notin S, \\ 1 / 2^{m} & \text { if } x \notin S \text { and } y=1 / 2^{m}, \\ |x-y| & \text { if } x, y \in S,\end{cases}
$$

where $S=\left\{1 / 2^{k}: k \in \mathbb{N}\right\}$. The topology induced by $p$ has as a base all sets of the form $\left\{1 / 2^{k}\right\}$ and $(X-S) \cup\left\{1 / 2^{k}: k \geq k_{0}\right\}$. Clearly $X$ is sequentially compact. The operator $T$ on $X$ defined by

$$
T(x)= \begin{cases}x, & \text { if } x \notin S, \\ x / 2, & \text { if } x \in S\end{cases}
$$

is continuous and $p\left(T(x), T^{2}(x)\right)<p(x, T(x))$ for $x \neq T(x)$. Hence by the corollary to Theorem $3, T$ has a fixed point, which however is not unique. It may be noted that though $T$ is a Banach operator of type $1 / 2$, Theorem 1 of [12] cannot be used here as $X$ is not Hausdorff.

The next result gives sufficient conditions under which the sequence of iterates of $T$ at a point converges. It is a generalization to gauge spaces of a result of Ortega and Rheinboldt [7, Section 12.3.5] for euclidean spaces and subsumes Sehgal's extension [11, Theorem 5] of Edelstein's earlier result [5, Theorem 1].

THEOREM 4. Let $T$ be a continuous operator on the gauge space $(X, \mathscr{P})$ into itself. Suppose that

(i) $p\left(T(x), T^{2}(x)\right) \leq p(x, T(x))$ for each $p \in P$;

(ii) if $x \neq T(x)$, then for each $p$ there exists an integer $n$ (which may depend on $x$ and $p)$, such that $p\left(T^{n}(x), T^{n+1}(x)\right)<p(x, T(x))$;

(iii) the closure, $S$, of $\left\{T^{n}\left(x_{0}\right): n=1,2, \ldots\right\}$ is sequentially compact, for some point $x_{0} \in X$;

(iv) $T$ has at the most one fixed point.

Then $\left\{T^{n}\left(x_{0}\right)\right\}$ converges to the unique fixed point of $T$.

Proof. That any limit point of $\left\{T^{n}\left(x_{0}\right)\right\}$ is a fixed point of $T$ is a consequence of Theorem 3 above. Since $T$ has at most one fixed point and $S$ is sequentially compact, $T$ has a unique fixed point $u$. For each $p \in \mathscr{P}$, 
$p\left(u, T^{n}\left(x_{0}\right)\right)$ converges to zero. Otherwise we can find a subsequence $y_{k}$ of $\left\{T^{n}\left(x_{0}\right)\right\}$ converging (in view of the sequential compactness of $S$ ) to some element $v$ and with the property that for some $p \in \mathscr{P}, p\left(u, y_{k}\right) \geq \varepsilon>0$ for a fixed real number $\varepsilon$. Proceeding to the limit we have that $p(u, v) \geq \varepsilon$. Thus $(v \neq u)$ would be a fixed point of $T$, contradicting that $u$ is the only fixed point.

Corollary (Sehgal [11]). If $(X, d)$ is a metric space and $T$ is a continuous operator on $X$ such that for $x \neq y, d(T(x), T(y))<\max \{d(x, T(x))$, $d(y, T(y)), d(x, y)\}$ then $T$ has a fixed point provided the sequence of $T$ iterates at $x_{0}$ has a convergent subsequence.

The deduction of the corollary rests on observing from the proof in [11] that $\left\{T^{n}\left(x_{0}\right)\right\}$ itself is convergent and so the closure $S$. of $\left\{T^{n}\left(x_{0}\right)\right\}$ is sequentially compact.

\section{References}

[1] S. Banach, 'Sur les opérations dans les ensembles abstraits et leur application aux équations intégrales', Fund. Math. 3 (1922), 133-181.

[2] D. F. Bailey, 'Some theorems on contractive mappings', J. London Math. Soc. 41 (1966), 101-106.

[3] J. Dieudonné, Foundations of Modern Analysis, Academic Press, New York (1962).

[4] M. Edelstein, 'An extension of Banach's Contraction principle', Proc. Amer. Math. Soc. 12 (1961), 7-10.

[5] M. Edelstein, 'On fixed and periodic points under contractive mappings', J. London Math. Soc. 37 (1962), 74-79.

[6] R. Kannan, 'Some results on fixed points', Bull. Calcutta Math. Soc. 60 (1968), 71-76.

[7] J. M. Ortega and W. C. Rheinboldt, Iterative solutions of non-linear equations in several variables, Academic Press, New York.

[8] S. Reich, 'Remarks on fixed points', Atti Acad. Naq. Lincei Rand. Sci. Fis. Mat. Natur. Serie VIII Vol. L11 face 5 (1972), 689-697.

[9] I. L. Reilly, 'Quasi-gauge spaces,' J. London Math. Soc. (2) 6 (1973), 481-487.

[10] I. L. Reilly, 'A generalized contraction principle', Bull. Austral. Math. Soc. 10 (1974), 359-363.

[11] V. M. Sehgal, 'On fixed and periodic points for a class of mappings', J. London Math. Soc. (2) 5 (1972), 571-576.

[12] P. V. Subrahmanyam, 'Remarks on some fixed point theorems related to Banach's contraction principle,' J. Math. Phys. Sci. 8 (1974), 445-457.

[13] K. K. Tan, 'Fixed point theorems for non-expansive mappings', Pacific. J. Math. 41 (1972), 829-842.

[14] A. Wilansky, 'Between $T_{1}$ and $T_{2}$ ', Amer. Math. Monthly 74 (1967), 261-266. 
Department of Mathematics

Indian Institute of Technology

Madras 600036

India
Department of Mathematics and Statistics University of Auckland Auckland New Zealand 